\title{
The development of unlabeled probes-high resolution melting (UP-HRM) marker on SAD, IAA27 and ACC genes of oil palm
}

\author{
TENGKU IMAM SAPUTRA ${ }^{*}$, ROBERDI, YOGO ADHI NUGROHO, WULAN ARTUTININGSIH, \\ OLIVIA S. PURBA, SIGIT DWI MARYANTO, DWI YONO, CONDRO UTOMO, TONY LIWANG
}

Department of Biotechnology, Plant Production and Biotechnology Division, PT SMART Tbk. Jl. Raya Cijayanti, Babakan Madang, Bogor 16810, West Java, Indonesia. Tel: +62-21 392 5720, `email: biotechnology@ sinarmas-agri.com.

Manuscript received: 31 March 2021. Revision accepted: 25 May 2021.

\begin{abstract}
Saputra TI, Roberdi, Nugroho YA, Artutiningsih W, Purba OS, Maryanto SD, Yono D, Utomo C, Liwang T. 2021. The development of unlabeled probes-high resolution melting (UP-HRM) marker on SAD, IAA27 and ACC genes of oil palm. Biodiversitas 22: 3356-3362. The unlabeled probes-high resolution melting (UP-HRM) marker is a useful tool for detecting of single nucleotide polymorphisms (SNPs). The objectives of this study were to develop UP-HRM markers to differentiate specific SNPs patterns on oil palm. The marker was developed and tested with Elaeis guineensis (Eg), Elaeis oleifera (Eo), Eo x Eg (hybrid), and was validated with 53 individuals of BC1F1 populations ((Eo x Eg) x Eg). Four UP-HRM markers were developed based on 2 SNPs in the stearoyl-acylcarrier-protein 9-desaturase (EgSAD), $1 \mathrm{SNP}$ in the auxin-responsive protein IAA27-like (EgIAA27), and 1 SNP in the 1-amino cyclopropane-1-carboxylate oxidase (EgACC) genes. The SNP discovery result showed that Eg was represented a reference homozygote genotype, while Eo was represented as an alternative homozygote genotype and the Eo x Eg hybrid was represented as a heterozygote genotype in all genes. The typical UP-HRM melt curve graph was successfully generated. This result was consistent with each genotype model for all four markers. The UP-HRM markers can distinguish each genotype according to the single-pass sequencing results. Furthermore, dendrogram analysis on validation divided $53 \mathrm{BC} 1 \mathrm{~F} 1$ samples into three cluster groups.
\end{abstract}

Keywords: HRM, interspecific hybrid, molecular markers, oil palm, SNPs genotyping

\begin{abstract}
Abbreviations: UP-HRM: The unlabeled probes-high resolution melting; SNPs: Single nucleotide polymorphisms; Eg: Elaeis guineensis; Eo: Elaeis oleifera, hybrid: Eo x Eg; BC1F1: Interspecific hybrid backcross; BC: Backcross; SAD: Stearoyl-acyl-carrierprotein 9-desaturase; IAA27: Auxin-responsive protein IAA27-like; ACC: 1-amino cyclopropane-1-carboxylate oxidase; CAPS: cleaved amplified polymorphic sequence
\end{abstract}

\section{INTRODUCTION}

Oil palms are classified into two species, which are Elaeis guineensis $(\mathrm{Eg})$ native to Africa and Elaeis oleifera (Eo), native to America (Montúfar et al. 2018). Eg is more productive in oil production than Eo and thus was planted commercially to fulfill the world vegetable oil demand. However, these two species are important germplasm for oil palm breeding program. Each of them has its superior characteristics. Oil palm breeding programs are starting to develop other added value characters such as higher proportion of unsaturated fatty acids, low height increment, canopy growth, resistance to diseases, tolerance to drought and low lipase activity after harvest (Turnbull et al. 2016; Lieb et al. 2017). These characters are naturally possessed by Eo.

As a perennial crop, oil palm breeding needs at least five to six backcross generations to obtain the progeny with recurrent parent genetic background with the transfer of the superior traits from the donor parent. Oil palm breeding takes 8-10 years per breeding cycle. However, molecular marker technology, such as marker-assisted selection (MAS) based on SNPs, can be applied to accelerate the breeding process for each trait of interest (Cobb et al. 2019). SNPs are important molecular markers for genotyping and alleles detection. They constitute the fundamental data of many heritability studies and breeding programs. Several methods have been developed to determine SNP genotyping including array-based hybridization, polymerase chain reaction (PCR), and sequencing. Array-based hybridization and sequencing require higher costs. Array-based hybridization is classified as commercial manufacturing, so it is less flexible, especially for small number of tested samples. Sequencing is a more efficient technique for SNPs discovery in an entire gene region. However, sequencing is considered redundant for data generated and costs incurred on genotyping of targeted SNPs that are already known. Thus, the PCR-based technique is considered more efficient for SNP genotyping with few numbers of samples.

Unlabeled Probe-high resolution melting (UP-HRM) is one of PCR-based genotyping technology. The advantage of UP-HRM method is not required fluorescent labels on their special primers or enzymes. UP-HRM is most costeffective compared to other genotyping technologies such as single-pass sequencing genotyping (Zhang et al. 2013; Lieveld et al. 2017) . UP-HRM is fast and strong, allowing rapid genotyping of many samples. The visualization shown is a variation of the melt curve based on doublestranded nucleotide hydrogen bond. It is categorized as an 
accurate and high-performance genotyping device (Lochlainn et al. 2011). HRM was developed on sweet cherry (Ganopoulos et al. 2013), rice (Zhu et al. 2013), cotton (Ghatage and Das 2016), and peanut (Kim et al. 2017). Moreover, HRM has been developed for rapid identification of genotypes and mutations induced by CRISPR/ Cas9 mutagenesis in rice (Li et al. 2018). In addition, HRM is commonly used for medical genotyping in humans, such as the identification of disease-related single nucleotide polymorphisms. For example, the HRM was used to detection of SNPs in genes associated with tumor necrosis factor (TNF) inhibitors efficacy (Yang et al. 2018), the sensitivity of warfarin in Chinese (Chen et al. 2014), preterm birth (Pereyra et al. 2012) and sickle cell disease (Yue et al. 2014).

The aim of this study was to develop a sensitive UPHRM marker to detect the genotype of Eg, Eo, Eo x Eg hybrid, and their $\mathrm{BC}_{1} \mathrm{~F}_{1}$ progeny using four SNPs at EgSAD (XM_010929403.3), EgSAD homolog (XM_029266457.1), EgIAA27 (XM_010919260.3) and EgACC (XM_010910523) genes. EgSAD gene is involved in oil synthesis (Montoya et al. 2014), IAA27 is correlated to somatic embryogenesis, the expression of IAA is decreased in response to increase of exogenous 2,4-D concentrations (Ooi et al. 2012). ACC is a gene responsible during the fruit developmental stage, it is an ethylene autoinhibitory system-1 (Tranbarger et al. 2011).

\section{MATERIAL AND METHODS}

\section{Genetic materials}

Two DNA samples from Elaeis guineensis (Eg), Elaeis oleifera (Eo) and Eo x Eg hybrid were used for the initial development stages of the genotype profiles which later became the control for the validation stages. Markers were tested with 53 BC1F1 samples. Genomic DNA was isolated using Isolate II Genomic DNA BIO-52066 (Bioline, USA) according to the guidelines. Quantity and quality of DNA were determined using UV-Vis NanoDrop ${ }^{\text {TM }}$ 2000/2000c Spectrophotometer (Thermo Fisher Scientific, USA) and $1 \%$ of agarose gel electrophoresis. The DNA concentration for amplification was diluted for $20 \mathrm{ng} \mu \mathrm{L}^{-1}$ and stored at $-20{ }^{\circ} \mathrm{C}$.

\section{In silico analysis}

In silico analysis was conducted to discover the SNPs in genes. All genes and chromosome sequence data of Eo were searched in the NCBI's Genbank database (https://www.ncbi.nlm.nih.gov/). Then the sequence data was re-validated using basic local alignment search tool (BLAST) analysis in the $\mathrm{Eg}$ genome database (http://genomsawit.mpob.gov.my). Whereas sequence data of Eo obtained by BLAST analysis using Eg sequence data compared to whole-genome shotgun contigs of Eo in https://blast.ncbi.nlm.nih.gov/Blast.cgi. Finally, all sequence data of genes and chromosomes were analyzed for multiple alignment and SNPs detection using Geneious Prime 2019.0.4 (Biomatters Ltd USA).

\section{PCR amplification}

Four pairs of primers and their unlabeled probes were designed to amplify the SNP target using the Primerquest tool (http://sg.idtdna.com/Primerquest/Home/Index) (Table 1). This study used normal forward and reverse primer pairs for amplification of DNA sequences. whereas the probes result from the Primerquest tool were modified by adding a different nucleotide base at the 3 'end to block the reaction without using a labeled reporter. Probe is a piece of DNA that is identical (or very similar) to the desired sequence or finds a specific DNA sequence containing SNP sites via hybridization.

The amplification was performed in a total volume of $17 \mu \mathrm{L}$ using MeltDoctor ${ }^{\mathrm{TM}}$ HRM Master Mix (Applied Biosystems, USA) and run through three-rounds thermal PCR profile. The first round of PCR was intended to amplify the target SNPs region. The PCR mix was consisted of 1x of MeltDoctor ${ }^{\mathrm{TM}}$ HRM Master Mix, $2 \mu \mathrm{M}$ of $\mathrm{MgCl}_{2}, 0.05 \mu \mathrm{M}$ of the forward primer, $0.5 \mu \mathrm{M}$ of reverse primer and $20 \mathrm{ng}$ of DNA template. The PCR reaction was run in a Thermal Cyclers SensoQuest (Sensoquest, Germany). Thermal profiles on PCR was started with 2 min of denaturation at $94^{\circ} \mathrm{C}$, followed by 55 cycles of $94^{\circ} \mathrm{C}$ for $30 \mathrm{sec}$, the annealing temperature appropriate for each primer in Table 1 for $30 \mathrm{sec}$, extension at $72^{\circ} \mathrm{C}$ for $40 \mathrm{sec}$, and incubation at $4^{\circ} \mathrm{C}$.

Table 1. Sequences of primers and unlabeled probes for the SNPs

\begin{tabular}{lllc}
\hline \multicolumn{1}{c}{ Marker name } & \multicolumn{1}{c}{ Primer } & \multicolumn{1}{c}{ Sequence (5'-3) } & Annealing temperature \\
\hline P5_126 & Forward & TCGAACTCCATCAAGTGTGTTA & 52 \\
& Reverse & AAGGAACTGAGAGAACGC & 56.5 \\
P13_124 & Probe & AGAGCTTCTTCAGTGATCATATCTCCc & 55 \\
& Forward & ACACCAAATGCAAGTAAGACCA & 60 \\
& Reverse & TGTAGGAAGGAATTGCTTCAGGTT & 52 \\
P11_137 & Probe & TCAAGAGCATGTCAAGAGCAGCATCc & 53 \\
& Forward & TACGTCCTTACATATGAAGACA & 56 \\
P3_146 & Reverse & ATCCTCACAACTACATCTTT & 5 \\
& Probe & TCTTCAGCAGATTTATGAATTTTATAGc & 5 \\
& Forward & CCCGAAGAAAGGCTCATGT & 5 \\
\hline
\end{tabular}

Note: *Underlined nucleotides denote the locations of the SNPs position 
The second round of PCR was carried out for melting acquisition. The first-round PCR product was added with $0.5 \mu \mathrm{M}$ of unlabeled probe primer. It allows the amplicon to react with an unlabeled probe primer to attach to the area SNP target. The reaction was run in Thermal Cycler with the following steps: early denaturation at $95{ }^{\circ} \mathrm{C}$ for $10 \mathrm{sec}$, annealing at $58^{\circ} \mathrm{C}$ for $20 \mathrm{sec}$, and incubation at $4^{\circ} \mathrm{C}$ for storage. The last PCR round was carried out for melting analysis in the Rotor-Gene Q (Qiagen, USA). Melting analysis was performed from $65^{\circ} \mathrm{C}$ to $90{ }^{\circ} \mathrm{C}$ with a slope of $0.15{ }^{\circ} \mathrm{C} / \mathrm{sec}$, giving 70 points $/{ }^{\circ} \mathrm{C}$. Melting curve data were analyzed using the Rotor-Gene Q software (Version 2.1.0, Qiagen, USA).

\section{High-resolution melting analysis}

UP-HRM melt curve data were obtained by melt analysis at a ramp of $10{ }^{\circ} \mathrm{C}$ with $0.1{ }^{\circ} \mathrm{C}$ increments for 2 sec. Fluorescent data were collected from each of the HRM temperature increased steps. Melting data was normalized by adjusting the start and end of fluorescence signals of all samples. UP-HRM melt curve data were recorded and analyzed using the Rotor-Gene 6500 series software (Corbett Research). HRM curve for each sample was scored. Different genotypes were identified by examining both normalized, difference, and derivative melt plots.

\section{DNA sequencing and UP-HRM confirmation}

Two samples each of Eg, Eo, and Eo x Eg hybrid with different HRM profiles were sequenced with single-pass DNA sequencing by the $1^{\text {st }}$ Base Laboratories, Singapore. Previously, PCR products were purified using a PCR Purification Kit (Qiagen, USA) following the standard protocol. Multiple alignments and SNPs detection was analyzed using Geneious Prime 2019.0.4 (Biomatters Ltd USA).

\section{Genetic distance and clustering}

Polymorphic markers were arranged as allelic data for genetic distance matrix estimation using a simple matching dissimilarity index. Based on this, an unweighted Neighbor-joining tree dendrogram (Saitou and Nei 1987) was computed and viewed using the Dissimilarity Analysis and Representation to Windows (DARwin5) version 6.0.8 (Perrier and Jacquemoud-Collet 2014). The robustness of branches was tested using 1.000 bootstraps. Polymorphism information content (PIC) and observed heterozygosity $\left(\mathrm{H}_{\mathrm{obs}}\right)$ were determined using Cervus software 2.0 (Marshall et al. 1998). Population structure analysis was performed to identify each probability value of the number of population clusters $(\mathrm{K})$ in a sample of individuals using STRUCTURE software ver. 2.3.4 (Pritchard et al. 2000). Then, the $\Delta \mathrm{K}$ was analyzed (Evanno et al. 2005).

\section{RESULTS AND DISCUSSION}

\section{SNP discovery}

SNP discovery was carried out by in silico analysis. The EgSAD gene (XM_010929403.3) and whole-genome shotgun contigs of Eo (ASIR01055092.1) were aligned to the reference genome of $\mathrm{Eg}$ chromosome 8 (NC_026000.1). Another EgSAD gene homolog (XM_029266457.1) and whole-genome shotgun contigs of Eo (ASIR01019242.1) were aligned to the reference genome of Eg chromosome 9 (NC_026001.1). EgACC gene (XM_010910523) and whole-genome shotgun contigs of Eo (ASIR01206136.1) were aligned to the reference genome of Eg genomic scaffold (NW_011559418.1). EgIAA27 gene (XM_010919260.3) and whole-genome shotgun contigs of Eo (ASIR01055276.1) were aligned to the reference genome of $\mathrm{Eg}$ chromosome 1 (NC_025993.1). SNP was found in all four genes. The SNPs of EgSAD, homolog EgSAD, EgACC and EgIAA27 were located at $8.307 .694 \mathrm{bp}, 6.281 .668$ bp. $2.728 \mathrm{bp}$ and 14.739.646 bp in particular chromosome. The SNP discovery showed that Eg was represented as a reference homozygote genotype, Eo was represented as an alternative homozygote genotype and the Eo $\mathrm{x}$ Eg hybrid was represented as a heterozygote genotype. The SNPs were used as a basis for UP-HRM primers design (Table 1).

The functional location and biological effects of all SNPs were in silico analyzed using Geneious Prime 2019.0.4 software (Biomatters Ltd USA). The analysis revealed that the SNPs on the EgSAD and EgSAD homolog were located in exon of chromosomes 8 and 9 . Both were synonymous SNPs, which means that they do not cause amino acid changes during translation. The SNP on EgIAA27 was located in the intron region, thus it has no effect on amino acid sequence during translation. SNPs in the intron region have also proved to be an interesting area for development as molecular markers, as shown by work done in rice (Badoni et al. 2016) and in Salvia miltiorrhiza (Wang et al. 2016). Furthermore, SNPs in EgACC were located in the exon region and non-synonymous, causes variations in the amino acid sequence during translation.

\section{The amplification and visualization of UP-HRM on Eo, Eg and Eo x Eg hybrid samples}

The four UP-HRM primer pairs were successfully amplified the DNA genome of Eo, Eg and Eo x Eg hybrid samples. UP-HRM development is based on asymmetric PCR concepts (Zhou et al. 2011). Many excessive single strands were hybridized with the unlabeled probe during asymmetric PCR. The SNP mismatch targets at the 3' of probes were blocked to prevent the extension reactions. Then the unlabeled probes in each primer pairs were produced a graphical visualization of the SNP effect. UPHRM amplicon was shown as melting curve peaks (Figure 1A). There were two melting curve peaks identified in UPHRM. The first melting curve peak was forward-reverse primer (FR) amplicon at higher melting temperature and the second was melting curve peak of the unlabeled probe at a lower melting temperature. Three melting curve motifs represented the three genotypes (Figure 1A). This observation indicated that allele differences (SNPs) were a factor that influenced the differences in the melting peak of each sample (Chou et al. 2020). 

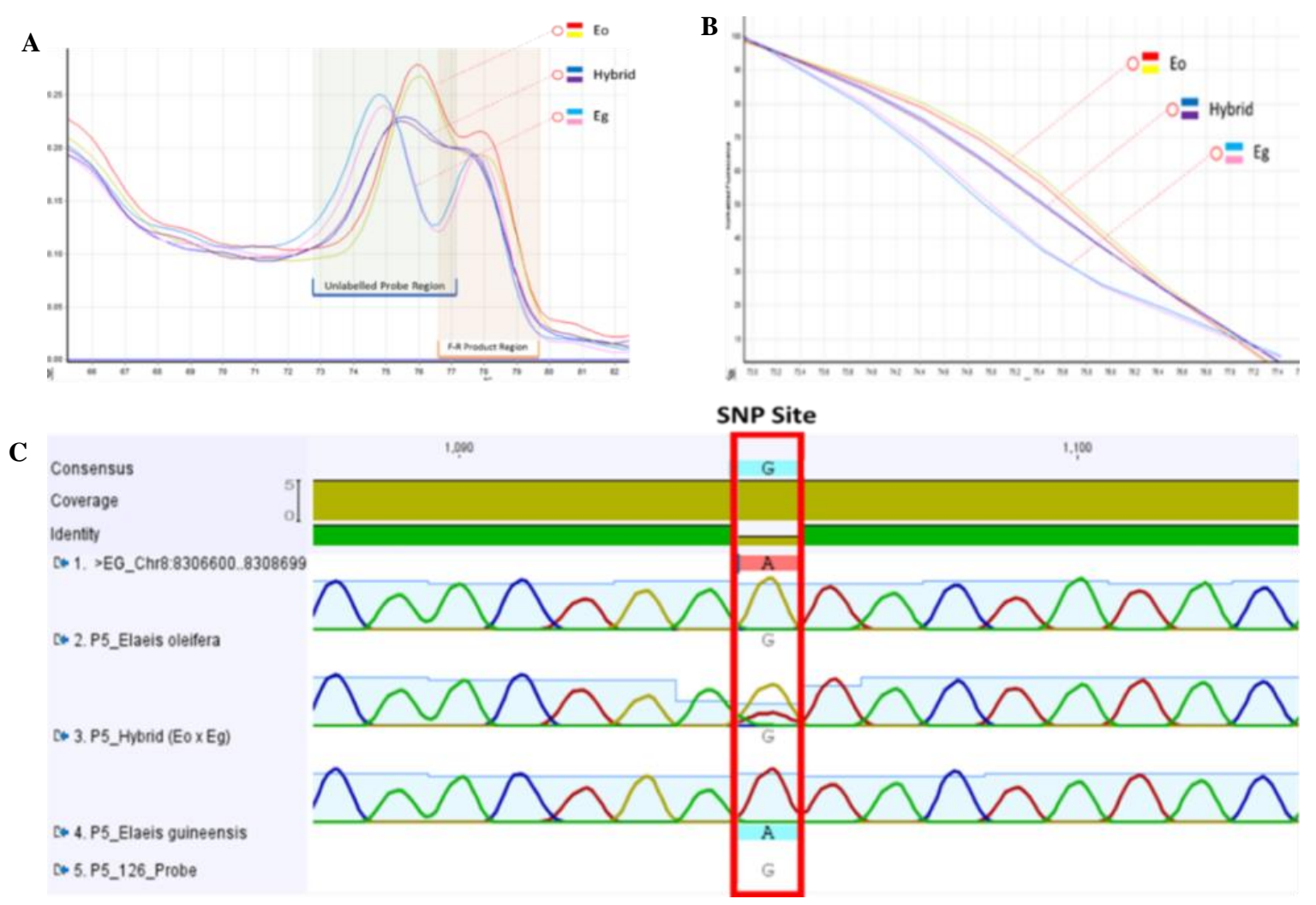

Figure 1. A: Melting profile of each 2 samples of Eg, Eo, and Eo x Eg hybrid on P5_126 primer. Unlabeled probe analysis was shown by a blue filter, while the result of the forward-reverse amplicon product was shown by the pink filter. B: Normalized melting curve. C): Single-pass sequencing analysis of all Eg and Eo samples, and Eo x Eg hybrid samples

The UP-HRM analysis is focused on the melting of the probe-target region. On P5_126 primer, it was observed between $72.8-77.2{ }^{\circ} \mathrm{C}$ and different temperatures were observed in the other primers. Eg samples were reached the melt curve peak faster than two other samples with a melting peak at $74.8^{\circ} \mathrm{C}$. All Eo samples were produced the slowest graph with a melting curve peak at $76^{\circ} \mathrm{C}$, and Eo $\mathrm{x}$ Eg hybrid samples have the melting curve peak in between Eg and Eo samples at $75.4{ }^{\circ} \mathrm{C}$. The melting curve was then normalized to facilitate genotype observation and to distinguish visually (Figure 1B). Subsequently, the UPHRM results were validated with single-pass sequencing to confirm all sample genotypes (Figure 1C).

Single-pass sequencing showed clear nucleotide electropherogram particularly in the SNPs target of all samples. Remarkably, all samples were grouped based on their corresponding melt curves. The Eg, Eo, and Eo x Eg hybrid samples showed the homozygous A-A, homozygous G-G genotype, and heterozygous A-G genotypes, respectively. The single-pass sequencing results were corresponding with the melting curve. The differences in melt curve peaks of all samples were caused by the hydrogen bond at the particular SNPs locus. The Eo reached a peak later than Eg caused by Eo contains G-G which has three hydrogen bonds that are more difficult to denature compared to AA (Eg) which only has two hydrogen bonds.
Thermal denaturation of double-stranded DNA (dsDNA) called DNA melting, depends on the length and the nucleotide sequence of dsDNA (Ruskova and Raclavsky 2011). Separation of dsDNA hydrogen bonds requires a certain amount of energy. G-C pair forms 3 hydrogen bonds, while the A-T pairs form two. Therefore, more $\mathrm{G}-\mathrm{C}$ in the molecules, requires more energy in the denaturation process than the A-T pairs. This provides a unique relationship between the length and nucleotide composition of dsDNA with their physical characteristics. The melting properties of dsDNA can be calculated by UV absorbance (hyperchromic effect). If two strands have the same sequence length but different GC content, the strand with less GC content will melt at the lower temperatures and this could be easily differentiated (Ririe et al. 1997). The differences in the melting curves, in particular the differences in the sequence of SNPs locations on dsDNA, can be analyzed using reference curves (the differences on curve peaks are based on known allele sequences). The samples were then grouped based on similar melting curves. If properly validated, the grouping of curves is reliable for SNPs identification (called a differential plot). HRM analysis can examine genotypes and mutation scanning (the discovery of new genetic variation) and also routine detection of known variations (Er and Chang 2012). 

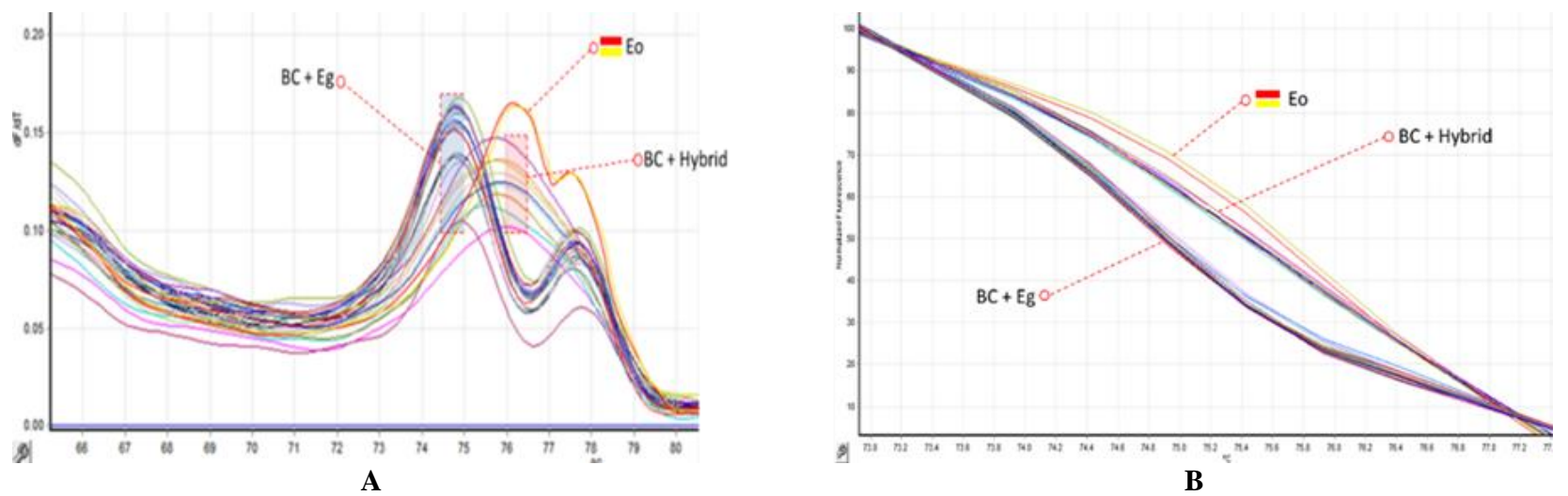

Figure 2. A: Melting profile of samples using primer P5_126. B: Normalized melting curve

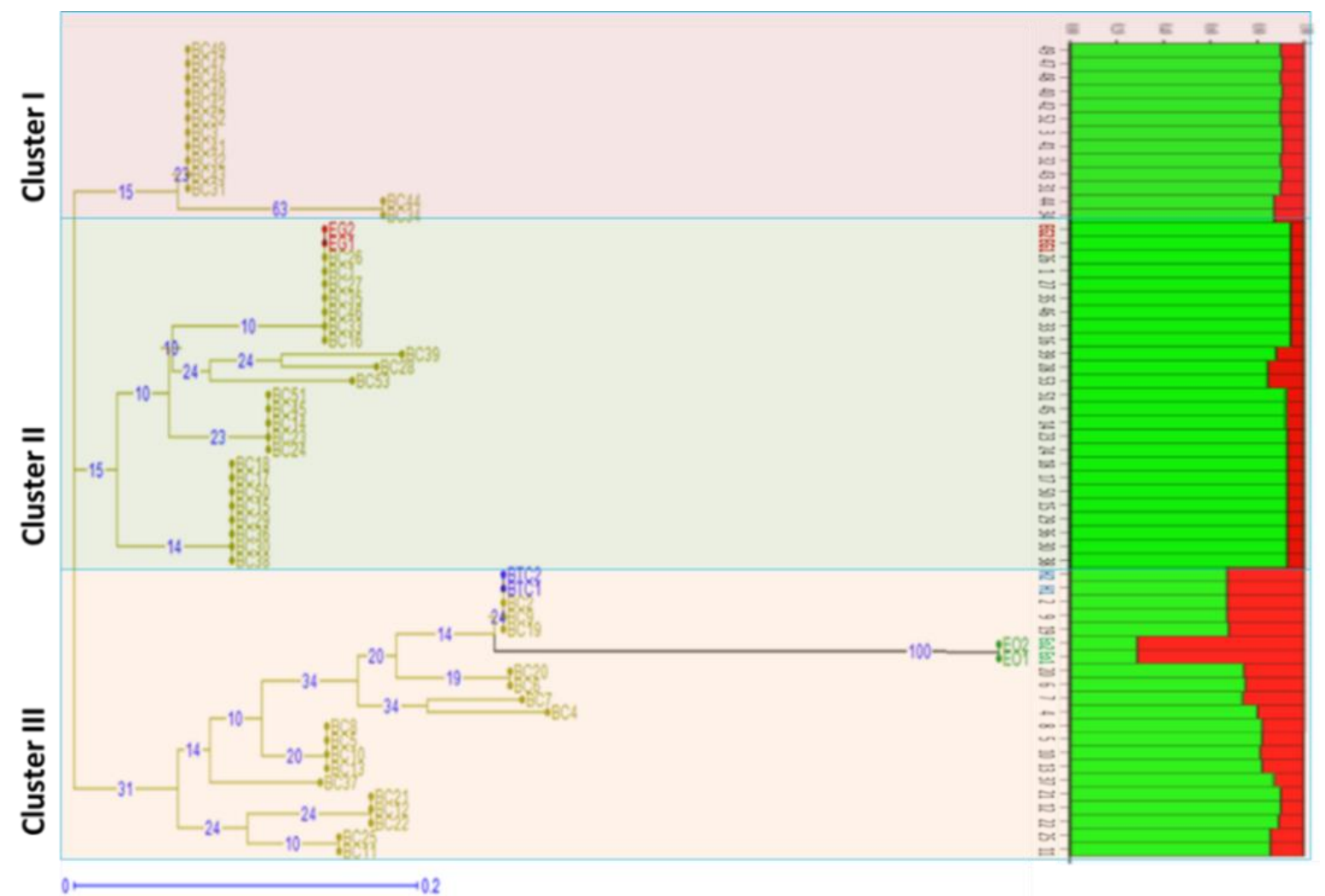

Figure 3. Dendrogram and Model-based simulation of population structure 59 accessions of oil palm. E. oleifera (EO/Green), E. guineensis (EG/Red), Eo x Eg hybrid (BTC/Blue); and backcross (BC/Brown)

\section{Validation with backcross (BC1F1) progeny}

Four UP-HRM markers were validated with 53 individual backcrosses (BC) progenies together with every two samples of Eo, Eg, and Eo x Eg hybrid as a positive control. All markers were produced polymorphic melting curves among all genotypes tested. Specific graphic patterns of Eg, Eo, Eo x Eg hybrid were grouped into three melt curve peaks based on their genotypes. All BC samples melt curves were grouped into Eg and Eo x Eg hybrid melt curve profiles (Figure 2). Genotype distribution of $53 \mathrm{BC}$ on P5_126 markers was AA (43 individual), AG (10 individual), and none of them with GG alleles. At P11_137 markers they were distributed as AA (21 individual), AC (32 individual), and $\mathrm{CC}$ (0 individual) and at P13_124 markers were AA (24 individual) or AC (29 individual), CC (0 individual). While at P3_146 markers they were distributed as CC (24 individual), CT (29 individual) and TT (0 individual).

Based on the analysis, all of UP-HRMs were polymorphic markers. Polymorphic Information Content (PIC) values were varied from 0.207 to 0.348 . The lowest PIC value was observed on the P5_126 loci, while the highest was at P13_124 loci (Table 2). PIC is explaining the marker polymorphism in the population, based on the number and frequency of alleles. The PIC describes the capacity of markers to distinguish species, depending on identified alleles and their frequency distribution, so that equal to genetic variation (Botstein et al. 1980). 
Table 2. Genetic diversity parameters of four loci primers

\begin{tabular}{|c|c|c|c|c|c|c|}
\hline Locus & Allele number & Allele & Allele frequency & H observed & H expected & PIC \\
\hline \multirow[t]{2}{*}{ P5_126 } & 2 & A & 0.86441 & 0.203 & 0.236 & 0.207 \\
\hline & & G & 0.13559 & & & \\
\hline \multirow[t]{2}{*}{ P13_124 } & 2 & A & 0.66102 & 0.542 & 0.452 & 0.348 \\
\hline & & $\mathrm{C}$ & 0.33898 & & & \\
\hline \multirow[t]{2}{*}{ P11_137 } & 2 & A & 0.70339 & 0.525 & 0.421 & 0.33 \\
\hline & & $\mathrm{C}$ & 0.29661 & & & \\
\hline \multirow[t]{2}{*}{ P3_146 } & 2 & $\mathrm{C}$ & 0.80508 & 0.322 & 0.317 & 0.265 \\
\hline & & $\mathrm{T}$ & 0.19492 & & & \\
\hline
\end{tabular}

\section{Distance-based dendrogram and population genetic structure}

Dendrogram analysis was constructed using the neighbor-joining method and simple matching distance matrix. Population structure was analyzed for a better understanding of the hierarchical population structure based on four UP-HRM marker loci. Based on the dendrogram analysis, oil palm samples were divided into three clusters (Figure 3). Thirteen backcross samples were clustered into cluster I. Two Eg and twenty-three backcross were in Cluster II and two Eo, two Eo x Eg hybrid and seventeen BC were in cluster III. Based on this BC19, BC9, BC2 will be conserved as parent candidates for further backcross program. Population structure analysis has supported the dendrogram. Fifty-three BC were represented by the proportion of two colors red and green (Figure 3). This color indicated the proportion of admixture alleles distribution of each accession.

In conclusion, this study demonstrated that UP-HRM markers could be developed and used in oil palm genotyping on EgSAD (XM_010929403.3), EgSAD homolog (XM_029266457.1), EgIAA27 (XM_010919260.3) and EgACC (XM_010910523) genes. All markers are considered as candidates for marker-assisted selection in the oil palm breeding program. This research demonstrates that UP-HRM can be used to precisely determine the presence of SNPs in oil palm. UP-HRM markers can be used to replace single-pass sequencing for targeted SNP genotyping when the number of samples is quite a large scale. This research should be continued with more markers and samples to determine genetic distances and the association between markers (genes) and phenotypes.

\section{ACKNOWLEDGEMENTS}

This work was funded by PT SMART Tbk under a research project code 3.1.1.023. We thank Ogi Ajitio Ramadhan and Redy Wahyu Permana for their technical supports. We thank Reno Tryono, Marcelinus R. Hatorangan, Victor Aprilyanto and Widyartini Made Sudania who read and improved the manuscript.

\section{REFERENCES}

Badoni S, Das S, Sayal YK, Gopalakrishnan S, Singh AK, Rao AR, Agarwal P, Parida SK, Tyagi AK. 2016. Genome-wide generation and use of informative intron-spanning and intron-length polymorphism markers for high-throughput genetic analysis in rice. Sci Rep 6: 1-15. DOI: 10.1038/srep23765.

Botstein, D, RL White, M Skolnick, RW Davis. 1980. Construction of a genetic linkage map in man using Restriction Fragment Length Polymorphisms. Am J Hum Genet 32 (3): 314-331.

Chen C, Li S, Lu X, Tan B, Huang C, Qin L. 2014. High-resolution melting method to detect single nucleotide polymorphism of VKORC1 and CYP2C9. Int J Clin Exp Pathol 7 (5): 2558-2564.

Chou, L Huang S-J, Hsieh C, Lu M-T, Song C-W, Hsu F-C. 2020. A high resolution melting analysis-based genotyping toolkit for the peach (Prunus persica) chilling requirement. Int J Mol Sci 21 (4): 1543. DOI: $10.3390 /$ ijms21041543.

Cobb JN, Biswas PS, Platten JD. 2019. Back to the future: Revisiting MAS as a tool for modern plant breeding. Theor Appl Genet 132 (3): 647-667. DOI: 10.1007/s00122-018-3266-4

Er T-K, Chang J-G. 2012. High-resolution melting: Applications in genetic disorders. Clin Chim Acta 414C: 197-201. DOI: 10.1016/j.cca.2012.09.012.

Evanno G, Regnaut S, Goudet J. 2005. Detecting the number of clusters of individuals using the software STRUCTURE: A simulation study. Mol Ecol 14 (8): 2611-2620. DOI: 10.1111/j.1365294X.2005.02553.x.

Ganopoulos I, Bazakos C, Madesis P, Kalaitzis P, Tsaftaris A. 2013. Barcode DNA high-resolution melting (Bar-HRM) analysis as a novel close-tubed and accurate tool for olive oil forensic use. J Sci Food Agric 93 (9): 2281-2286. DOI: 10.1002/jsfa.6040.

Ghatage TS, Das P. 2016. High resolution melting curve analysis: An efficient method for genetic purity analysis of cotton (Gossypium hirsutum) hybrid and their parental lines. IOSR J Biotechnol Biochem 2 (7): 49-53. DOI: 10.9790/264X-03027882.

Kim K-S, Lee D, Bae SB, Kim Y-C, Choi I-S, Kim ST, Lee T-H, Jun TH. 2017. Development of SNP-based molecular markers by resequencing strategy in peanut. Plant Breed Biotechnol 5 (4): 325-333. DOI: 10.9787/PBB.2017.5.4.325.

Li S, Liu S, Liu Y, Lu H, Tan Y, Huang J, Wei P, Shu QY. 2018. HRMfacilitated rapid identification and genotyping of mutations induced by CRISPR/ Cas9 mutagenesis in rice. Crop Breed Appl Biotechnol 18 (2): 184-191. DOI: 10.1590/1984-70332018v18n2a26.

Lieb VM, Kerfers MR, Kronmüller A, Esquivel P, Alvarado A, Jiménez VM, Schmarr H, Carle R, Schweiggert RM, Steingass CB. 2017. Characterization of mesocarp and kernel lipids from Elaeis guineensis Jacq., Elaeis oleifera [Kunth] Cortés, and their interspecific hybrids. J Agric Food Chem 65 (18): 3617-3626. DOI: 10.1021/acs.jafc.7b00604.

Lieveld M, Carregosa A, Benoy I, Redzic N, Berth M, Broeck DV. 2017. A high resolution melting (HRM) Technology-based assay for costefficient clinical detection and genotyping of herpes simplex virus (HSV)-1 and HSV-2. J Virol Methods 248: 181-186. DOI: 10.1016/j.jviromet.2017.07.005 
Lochlainn SÓ, Amoah S, Graham NS, Alamer K, Rios JJ, Kurup S, Stoute A, Hammond JP, Østergaard L,King GJ, White PJ, Broadley MR. 2011. High-resolution melt (HRM) analysis is an efficient tool to genotype EMS mutants in complex crop genomes. Plant Methods 7 (1): 43. DOI: 10.1186/1746-4811-7-43.

Marshall TC, Slate J, Kruuk LE, Pemberton JM. 1998. Statistical confidence for likelihood-based paternity inference in natural populations. Mol Ecol 7 (5): 639-655. DOI: 10.1046/j.1365294x.1998.00374.x.

Montoya C, Cochard B, Flori A, Cros D, Lopes R, Cuellar T, Espeout S, Syaputra I, Villeneuve P, Pina M, Ritter E, Leroy T, Billotte N. 2014 Genetic architecture of palm oil fatty acid composition in cultivated oil palm (Elaeis guineensis Jacq.) compared to its wild relative $E$. oleifera (H.B.K) Cortés. PLoS ONE 9 (5): e95412. DOI: 10.1371/journal.pone.0095412.

Montúfar R, Louise C, Trabarger T. 2018. Elaeis oleifera (Kunth) Cortés: A neglected palm from the Ecuadorian Amazon. Rev Ecuat de Med y Cienc Biol 39 (1): 10-18. DOI: 10.26807/remcb.v39i1.584.

Ooi SE, Choo CN, Ishak Z, Ong-Abdullah M. 2012. A candidate auxinresponsive expression marker gene, EgIAA9, for somatic embryogenesis in oil palm (Elaeis guineensis Jacq.). Plant Cell Tiss Organ Cult 110: 201-212. DOI: 10.1007/s11240-012-0143-8.

Pereyra S, Velazquez T, Bertoni B, Sapiro R. 2012. Rapid multiplex high resolution melting method to analyze inflammatory-related SNPs in preterm birth. BMC Res Notes 5 (1): 69. DOI:10.1186/1756-0500-5 69.

Perrier X, Jacquemoud-Collet JP. 2014. DARwin Software (Dissimilarity Analysis Representation for Windows). http://darwin.cirad.frLast.

Pritchard JK, Stephens M, Donnelly P. 2000. Inference of population structure using multilocus genotype data. Genetics 155 (2): 945-959.

Ririe KM, Rasmussen RP, Wittwer CT. 1997. Product differentiation by analysis of DNA melting curves during the polymerase chain reaction. Anal Biochem 245 (2): 154-160. DOI: 10.1006/abio.1996.9916.

Ruskova L, Raclavsky V. 2011. The potential of high resolution melting analysis (HRMA) to streamline, facilitate and enrich routine diagnostics in medical microbiology. Biomed Pap Med Fac Univ
Palacky Olomouc Czech Repub 155 (3): 239-252. DOI: $10.5507 / \mathrm{bp} .2011 .045$

Saitou N, Nei M. 1987. The neighbor-joining method: A new method for reconstructing phylogenetic trees. Mol Biol Evol 4 (4): 406-425. DOI: 10.1093/oxfordjournals.molbev.a040454.

Tranbarger TJ, Dussert S, Joët, T Argout X, Summo M, Champion A, Cros D, Omore, A Nouy B, Morcillo F. 2011. Regulatory mechanisms underlying oil palm fruit mesocarp maturation, ripening, and functional specialization in lipid and carotenoid metabolism. Plant Physiol 156 (2): 564-584. DOI: 10.1104/pp.111.175141.

Turnbull NC, Guerin M, Louise C, Amblard C, Cochard P, DurandGasselin T. 2016. Oil palm breeding and seed production in Africa. Proceedings of the International Seminar on Oil Palm Breeding and Seed Production and Field Visits (September): 21-38.

Wang H, Hao N, Chen L, Li G. 2016. Development of intron polymorphism markers in major latex-like protein gene for localitylevel and cultivar identification of Salvia miltiorrhiza. Springerplus 5 (1): 1919. DOI:10.1186/s40064-016-3611-5.

Yang, M-J, Hou Y, Yan X-L, Wang C-X, Zhi L-X, You C-G. 2018. Development and application of a PCR-HRM molecular diagnostic method of SNPs linked with TNF inhibitor efficacy. Diagnosis 6 (3): 277-286. DOI:10.1515/dx-2018-0062.

Yue, Liang et al. 2014. Rapid screening for sickle cell disease by polymerase chain reaction-high resolution melting analysis. Mol Med Rep 9 (6): 2479-2484. DOI: 10.3892/mmr.2014.2130.

Zhang L, Cui G, Li Z, Wang H, Ding H, Wang DW. 2013. Comparison of high-resolution melting analysis, taqman allelic discrimination assay, and sanger sequencing for clopidogrel efficacy genotyping in routine molecular diagnostics. J Mol Diagn 15 (5): 600-606. DOI: 10.1016/j.jmoldx.2013.04.005.

Zhou L, Wang Y, Wittwer CT. 2011. Rare allele enrichment and detection by allele-specific PCR, competitive probe blocking, and melting analysis. BioTechniques 50 (5): 311-318. DOI: 10.2144/000113668.

Zhu Y, Wang Q, Hu J, Zhu L, Wang J, Zhu S, Guan Y. 2013. High resolution melting curve analysis: An efficient method for fingerprinting of hybrid rice cultivars and their parental lines. Australian J Crop Sci 7 (13): 2048-2053. 